\title{
Ultrasonic Pulse-Echo Subwavelength Defect Detection Mechanism: Experiment and Simulation
}

\author{
Xiangtao Yin, ${ }^{1}$ Scott A. Morris, ${ }^{2}$ and William D. O'Brien, Jr., ${ }^{1,3}$
}

Received March 5, 2003; revised September 22, 2003

\begin{abstract}
The ultrasonic pulse-echo backscattered amplitude integral (BAI)-mode imaging technique [IEEE Trans. UFFC, 45:30 (1998)] has demonstrated sensitive detection of subwavelength channel defects (38- $\mu \mathrm{m}$ diameter reliably and $6-\mu \mathrm{m}$ diameter occasionally) in flexible $220-\mu \mathrm{m}$-thick food package seals $(17.3 \mathrm{MHz}, \lambda \approx 86 \mu \mathrm{m})$. However, the underlying subwavelength defect detection mechanism is poorly understood. In this contribution, a theoretical modeling study was undertaken to elucidate the mechanism. The subwavelength diameter channel was fused in-between two plastic package films by applying heat from one side of the films. The sample cross-section microstructure was characterized from both optical and acoustic images. The cross-section impedance profiles along sample thickness dimension were determined. Although identical in nominal impedance properties before sealing, the two binding films showed an asymmetric impedance profile after sealing. Transient finite-element heat conduction analysis and impedance profiles of multiple-sealed package samples showed that the single-sided heating process caused an asymmetric impedance profile. A generalized impedance model was proposed based on these observations. An efficient two-dimensional simulation tool using a finite-difference time-domain method and the perfectly matched layer numerically evaluated the defect detection behavior of the radio-frequency (rf) echo waveforms. The normalized correlation coefficients between the simulated and the measured rf echo waveforms were greater than $95 \%$ for this generalized model, which suggested the validity of the proposed impedance model.
\end{abstract}

KEY WORDS: Channel defect; material characterization; plastic seals; pulse-echo detection; ultrasonic nondestructive evaluation (NDE).

\section{INTRODUCTION}

Ultrasonic pulse-echo techniques have popular applications in nondestructive evaluation, material

${ }^{1}$ Bioacoustics Research Laboratory, Department of Electrical and Computer Engineering, University of Illinois at Urbana-Champaign, Urbana, Illinois 61801.

${ }^{2}$ Packaging Program, Department of Food Science and Human Nutrition, University of Illinois at Urbana-Champaign, Urbana, Illinois 61801

${ }^{3}$ Address correspondence to William D. O'Brien, Bioacoustics Research Laboratory, Department of Electrical and Computer Engineering, University of Illinois at Urbana-Champaign, Urbana, Illinois 61801. E-mail:wdo@uiuc.edu characterization, and biomedical images. The ultrasonic pulse-echo backscattered amplitude integral (BAI)mode imaging technique has been developed to detect small channel defects embedded in flexible food package seals. ${ }^{(1)}$ The BAI-mode image is constructed from the BAI values; values that are determined at each transducer sampling position by taking the area under the absolute magnitude of the envelope of the received backscattered echo. This technique has demonstrated sensitive subwavelength defect detection capability; for example, $100 \%$ detection of water-filled channels (cylindrical channels in bonded two-sheet plastic package samples, thickness $110 \mu \mathrm{m}$ per sheet) as small as $38 \mu \mathrm{m}$ in diameter with a $17.3-\mathrm{MHz}$ focused (f/2) 
transducer in water $\left(20^{\circ} \mathrm{C}, c \approx 1485 \mathrm{~m} / \mathrm{s}, \lambda \approx 86 \mu \mathrm{m}\right)$. Moreover, water-filled channel defects as small as $6 \mu \mathrm{m}$ in diameter could intermittently be detected. ${ }^{(2)}$

The BAI-mode image pixel values are interpolated from the BAI values at each transducer scan location. The average BAI-value difference between the defective and intact regions of the package sample is a sensitive image contrast index with respect to channel defect size, material type inside channel defect, package material type, and transducer scanning step size. ${ }^{(2-6)}$ The changes in the BAI values are indeed the indications of backscattered echo signal strength and variation.

Changes in the radio-frequency (rf) echo waveforms affect the image contrast. Two measured rf echo waveforms are displayed in Fig. 1. The solid line is the echo from the intact region. The dashed line is the echo from the 38- $\mu \mathrm{m}$-diameter channel. The two rf echoes have equal amplitude between round-trip times (RTT) of $16.82 \mu \mathrm{s}$ and $16.92 \mu \mathrm{s}$, which corresponds with specular reflection from the package front wall facing the transducer. The specular reflection from the package sample back wall appears at around $17.01 \mu \mathrm{s}$. The time interval of $(16.92,17.01) \mu s$ corresponds approximately with a $100-\mu \mathrm{m}$ distance. The average speed of sound in the plastic film is approximately $2500 \mathrm{~m} / \mathrm{s}$, and the time interval is the pulse-echo round-trip time. Two interesting features have been observed in the time interval $(16.92,17.01) \mu s$. First, the rf echo from the intact region is not zero. Therefore, the specular reflection echoes from front and back walls are not well-separated in time. There may, however, be anomalous impedance differences in this region, but we believe that this is unlikely. Second, the channel defect has an obvious influence on the echo, suggesting echo amplitude changes due to the subwavelength channel defect. We therefore

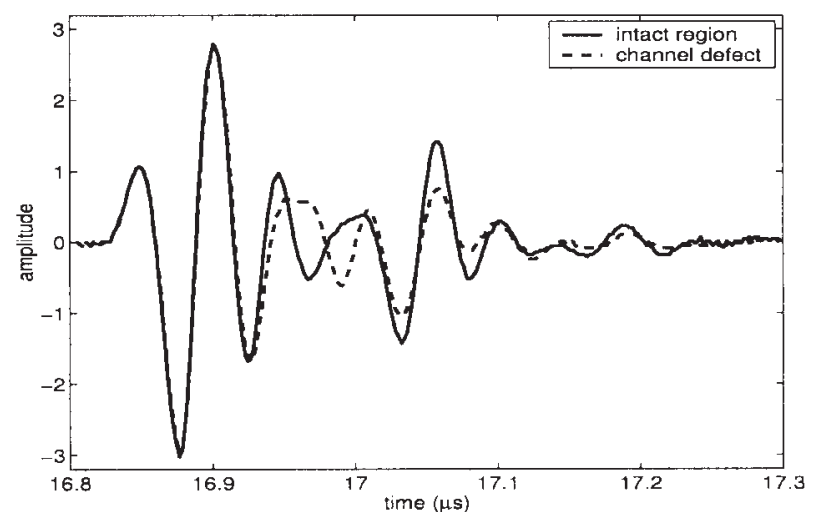

Fig. 1. Radio-frequency echo waveforms acquired in experiment (from a water-filled $38-\mu \mathrm{m}$-diameter channel defect in a $220-\mu \mathrm{m}$ thick plastic package, transducer frequency $17.3 \mathrm{MHz}$ ). are hypothesizing that the subwavelength channel defect affects the rf echo waveforms and thus the BAI values.

The BAI-mode image has been proven to be reliable for detecting subwavelength channel defects with high sensitivity; however, the underlying detection mechanism is poorly understood. The package sample has a stratified multilayer structure (see Sec. 3.1). The ultrasound beam encounters complex structures in its propagation path: the package sample front wall, interfaces between sublayers, heat-fusing zone between the two plastic sheets, the channel itself, impedance deviation surrounding the channel, and the package sample back wall. The water-filled subwavelength channel in a thin stratified package sample represents a small geometrical region with a different impedance relative to its surrounding medium. Such an obstacle or inhomogeneity in the path of a sound wave causes scattering because the obstacle interacts with the incident wave. ${ }^{(7)}$ However, as the region is subwavelength in scale, the backscattered echo strength will be very weak compared with the specular reflection from the large flat interfaces between package sample and water. Furthermore, if one considers system noise and multiple reflections caused by package sublayer interfaces, then such a small perturbation in the stratified medium might tend to be too weak to affect significantly an ultrasonic field.

A theoretical modeling study was undertaken to investigate the origin of the BAI-mode image contrast mechanism. A generalized impedance model was proposed through experimental characterization of the package sample cross section. Because it was difficult to control the channel defect package sample microstructure during the heat-sealing process, a numerical evaluation procedure was used rather than experimentally examining the proposed impedance model. An efficient two-dimensional (2D) numerical simulation tool using a finite-difference time-domain (FDTD) method with the perfectly matched layer (PML) absorbing boundary ${ }^{(8-10)}$ was developed to evaluate the aforementioned pulse-echo interactions between the ultrasound beam and sample microstructure. FDTD is good at simulating transient wave propagation and scattering phenomena. ${ }^{(1-14)}$ The PML absorbs the outgoing wave at the truncated computational domain so that a simulated free space can be achieved. ${ }^{(10,15)}$ The study presented in this contribution aims at a better understanding of the fundamental ultrasonic pulse-echo subwavelength defect detection mechanism through both experimental and numerical modeling approaches. 


\section{NUMERICAL SIMULATION}

Using FDTD with a PML, simulation of the ultrasonic pulse-echo defect detection behavior was implemented by solving the 2D linear acoustic wave equation in a homogeneous, lossy fluid medium given by

$$
\begin{aligned}
& \nabla p(\mathbf{r}, t)=-\rho \frac{\partial}{\partial t} \mathbf{u}(\mathbf{r}, t)-\alpha^{*} \mathbf{u}(\mathbf{r}, t) \\
& \nabla \cdot \mathbf{u}(\mathbf{r}, t)=-\kappa \frac{\partial}{\partial t} p(\mathbf{r}, t)-\alpha p(\mathbf{r}, t)
\end{aligned}
$$

where $p$ is the acoustic pressure field, $\mathbf{u}$ is the vector velocity field, $\mathbf{r}$ is the vector field point, $t$ is time, $\rho$ is the mass density of the medium, $\kappa$ is the compressibility of the medium, $\alpha$ is the absorption coefficient associated with compressibility, and $\alpha^{*}$ is the absorption coefficient associated with mass density. As an artificial absorbing material layer, the PML surrounds the acoustic medium region in the FDTD simulation domain. By separating the acoustic pressure field mathematically and constructing an anisotropic attenuation coefficient $\alpha^{*}$, the PML has the same characteristic impedance as that of the acoustic medium yet significantly absorbs the incident acoustic wave with little reflection. The absorption in the PML is achieved by setting $\alpha^{*}=\alpha \rho / \kappa$ in the PML region and $\alpha^{*}=0$ in the acoustic medium. ${ }^{(10)}$

The simulation adopts the transducer parameters (center frequency $17.3 \mathrm{MHz}$, diameter $6.35 \mathrm{~mm}$, focal length $12.44 \mathrm{~mm}$ ) in order to compare the simulated rf echo waveforms with the measured waveforms (Fig. 1) acquired by the spherically focused transducer (model V317, GE Panametrics, Waltham, MA). However, it is prohibitive to include the transducer ( $74 \lambda$ wide) and the entire transducer-to-sample wave propagation path (145 $\lambda$ long) in the FDTD computational domain due to impractical computational cost and large dispersion errors. ${ }^{(15-17)}$

Two techniques are used to reduce the computational domain. First, FIELD-II ${ }^{(18)}$ is used to calculate the equivalent ultrasound pulse pressure field transmitted to the line $A B$ in Fig. 2. The transmitted pulse in the simulation is described as ${ }^{(19)}$

$$
p_{\text {inc }}(t)=\frac{t}{T} \exp \left(-4 \Delta f^{2} t^{2}\right) \sin \left(2 \pi f_{0} t\right)
$$

where $f_{0}=17.3 \mathrm{MHz}$ is the transducer center frequency, $T=2 / f_{0}$ is the pulse duration of the transmitted pulse, and $\Delta f=7.35 \mathrm{MHz}$ is the $-3-\mathrm{dB}$ transducer bandwidth. Second, the rf echo waveform received by the transducer surface is extrapolated by evaluating the transient Helmholtz integral

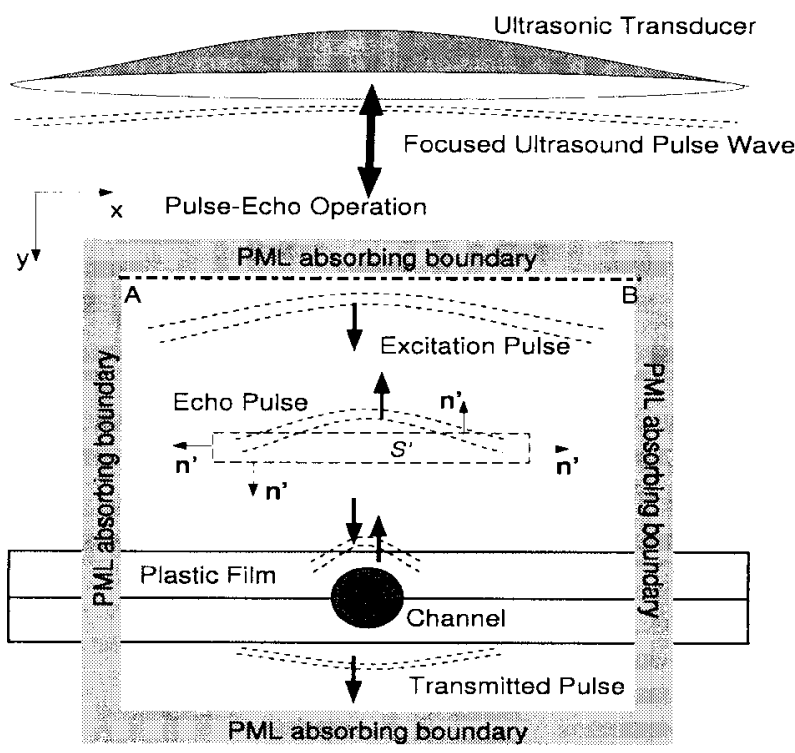

Fig. 2. Pulse-echo simulation with FDTD and PML.

$p(\mathbf{r}, t)=\frac{1}{2 \pi} \oint_{s^{\prime}}\left[\frac{\partial r}{\partial \mathbf{n}^{\prime}}\left(\frac{\tilde{p}\left(t-\frac{r}{c}\right)}{r^{2}}+\frac{1}{c r} \frac{\partial \tilde{p}\left(t-\frac{r}{c}\right)}{\partial t}\right)+\frac{1}{r} \frac{\partial \tilde{p}\left(t-\frac{r}{c}\right)}{\partial \mathbf{n}^{\prime}}\right] \mathrm{d} S^{\prime}$

along the enclosed contour $S^{\prime}$ in Fig. $2,{ }^{(20,21)}$ where $\mathbf{n}^{\prime}$ is the outward surface norm on $S^{\prime}$ and $c$ is the speed of sound in the medium. The enclosed surface $S^{\prime}$ consists of evenly divided radiating elements with locations $\left(x^{\prime}, y^{\prime}\right)$. The transducer surface is partitioned with equally sized receiving elements with locations $(x, y)$. The distance between each radiating-receiving pair is $\boldsymbol{r}=\hat{\imath}\left(x-x^{\prime}\right)+\hat{j}\left(\mathrm{y}-y^{\prime}\right)$. The pressure $\tilde{\mathrm{p}}\left(t-\frac{r}{c}\right)$ on $S^{\prime}$ is computed by the FDTD simulation at each time step. The computational domain is significantly reduced from $145 \lambda$ long by $47 \lambda$ wide to $10 \lambda$ by $10 \lambda$ with FIELD-II and the transient Helmholtz integral.

The 2D simulation using FDTD and PML in Cartesian coordinates was implemented in MATLAB (The Math Works, Inc., Natick, MA). A spatialtemporal update scheme similar to the well-known Yee algorithm ${ }^{(8)}$ was adopted in the FDTD simulation. To ensure stability, the grid size $\triangle=\lambda_{\text {min }} / 20$ and the time evolution step $h=\triangle / 2 c_{\max }$, where $\lambda_{\min }$ is the smallest wavelength and $c_{\max }$ is the greatest speed of sound in the computational domain. The PML had a 10 -grid thickness.

Except for the routine validation tests for FDTD and PML implementation, the incorporation of FIELD-II and the transient Helmholtz integral [Eq. (3)] into the FDTD-PML code has been validated. A simulated transducer pulse-echo field characterization experiment $^{(22)}$ was implemented in MATLAB. The 
excitation signal was taken from Eq. (2). In the simulation, a $38-\mu \mathrm{m}$-diameter tungsten wire was placed in the computational domain to generate rf echo waveforms. The transducer and its waveform parameters were extracted by processing the simulated echo waveform data set. Comparisons among the experimental measurements, the FIELD-II simulation parameters, the simulated characterization results, and the calculated results from theory ${ }^{(22)}$ are shown in Table I. The simulation agrees with the experiment very well.

\section{PACKAGE SAMPLE MICROSTRUCTURE}

An impedance profile model had to be established for the pulse-echo subwavelength defect detection mechanism study. The impedance profile of interest is the package sample cross-section impedance map.

\subsection{Package Sample Preparation}

The package sample was produced by using a heat sealer (Audionvac-VM151HG, Audion Electro, Weesp, Holland) to fuse a smooth, die-drawn tungsten wire in-between two plastic trilaminate package films (Fuji Tokushu Shigyo Co. Ltd., Seto Aichi, Japan). Each trilaminate package film has three sublayers: polypropylene layer $\left(80 \mu \mathrm{m}, c=2660 \mathrm{~m} / \mathrm{s}, \rho=900 \mathrm{~kg} / \mathrm{m}^{3}\right)$, polyvinylidene chloride (PVDC) layer $(15 \mu \mathrm{m}, c=2380 \mathrm{~m} / \mathrm{s}$, $\left.\rho=1670 \mathrm{~kg} / \mathrm{m}^{3}\right)$, and oriented nylon layer $(15 \mu \mathrm{m}, c=$ $2600 \mathrm{~m} / \mathrm{s}, \rho=1140 \mathrm{~kg} / \mathrm{m}^{3}$ ). The inner layer (polypropylene) is the heat-sealing layer. The middle layer (PVDC) provides a barrier to chemical substances. The outer sublayer (nylon) gives strength, a barrier to gases, oils, and fats, and scuff resistance to the printed surface.

The heat sealer has a chamber with a heavy lid. Attached to the lid was a flat silicone rubber bar to apply uniform pressure on the package sample. Inside the chamber was a flat heating bar with a nonstick teflon coating. After closing the lid, the package sample was clamped between the flat rubber bar and the flat heating bar to ensure intimate contact of the two inner faces [Fig. 3(a)]. The chamber was first vacuumed to expel the air inside the package sample.

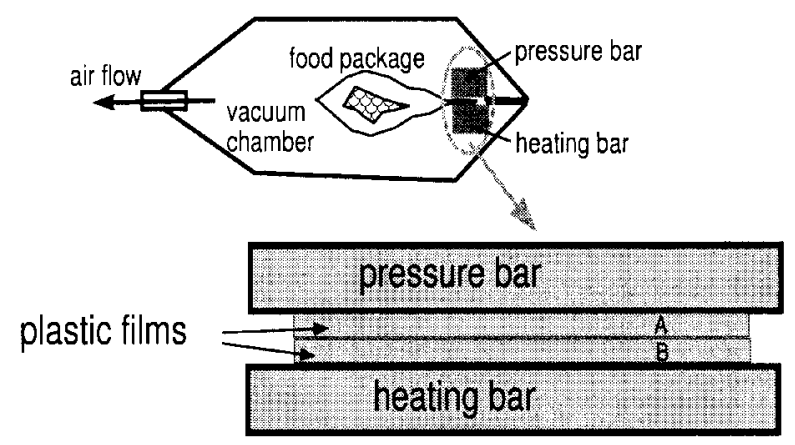

(a)

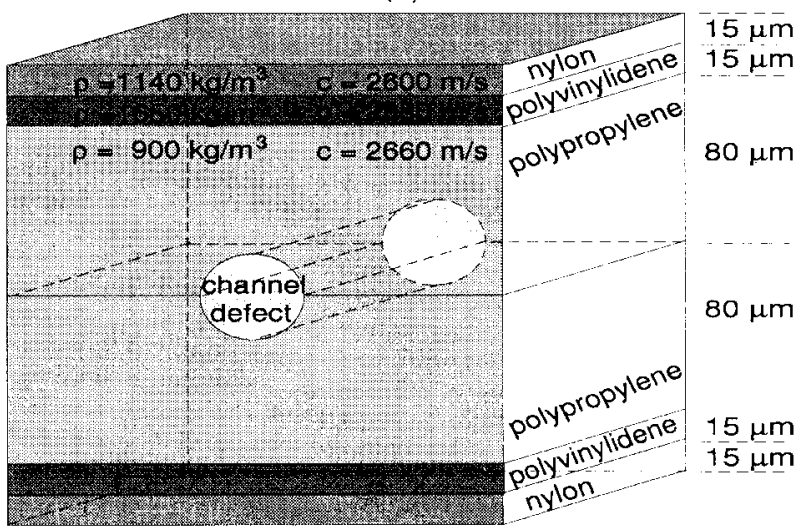

(b)

Fig. 3. (a) Illustration of the heat sealing process; (b) the channel defect sample structure.

Table I. Transducer Characteristics Obtained from Numerical Simulation

\begin{tabular}{|c|c|c|c|c|}
\hline & $\begin{array}{c}\text { Experiment } \\
\text { characterization }\end{array}$ & $\begin{array}{l}\text { Parameters } \\
\text { in FIELD-II }\end{array}$ & $\begin{array}{c}\text { Simulated } \\
\text { characterization }\end{array}$ & Theory \\
\hline$D(\mathrm{~mm})$ & 6.35 & 6.35 & 6.35 & 6.35 \\
\hline$f^{\#}$ & 1.96 & 2 & 1.98 & 2 \\
\hline$f_{0}(\mathrm{MHz})$ & 17.3 & 17.3 & 17.8 & 20.0 \\
\hline$\Delta f_{(-3 d B)}(\mathrm{MHz})$ & 7.35 & 7.35 & 8.12 & 5.95 \\
\hline Fractional BW & $43.5 \%$ & $43.5 \%$ & $34.8 \%$ & $27.4 \%$ \\
\hline$F_{L}(\mathrm{~mm})$ & 12.44 & 12.7 & 12.65 & 12.7 \\
\hline $\mathrm{RTT}(\mu \mathrm{s})$ & 16.8 & No input needed & $\begin{array}{c}17.0 \\
(c=1485 \mathrm{~m} / \mathrm{s})\end{array}$ & $\begin{array}{c}17.1 \\
(c=1485 \mathrm{~m} / \mathrm{s})\end{array}$ \\
\hline$F_{z}(\mathrm{~mm})$ & 2.15 & No input needed & 2.72 & 2.42 \\
\hline$B D_{(-6 d B)}(\mu \mathrm{m})$ & 173 & No input needed & 193 & 176 \\
\hline
\end{tabular}


Then the heating bar was heated to the designated temperature $\left(\sim 130{ }^{\circ} \mathrm{C}\right)$ within a user-specified amount of time. The heat was applied from the heating bar and passed through the sublayers. The inner sealant layers were melted and fused together. The sample was then cooled to room temperature.

To fabricate different-sized channel defects, tungsten wires with different diameters $(6,10,15,25,38$, 50 , or $75 \mu \mathrm{m}$ ) were sandwiched transversely to the sealing direction between two inner sublayers. After the sample had cooled, the tungsten wire was withdrawn along its axial direction from the fused plastic sample in degassed water to create a water-filled channel defect [Fig. 3(b)].

\subsection{Experimental Characterization}

The "pulse-echo reference measurement" procedure measures rf echo waveform, with the same equipment settings, from both a reference material plate whose impedance value is known and the unknown material whose impedance value is to be determined. A simple follow-up mathematical operation calculates the acoustic impedance value for the unknown material by taking the amplitude ratio between the two measurements. To measure the package sample impedance profile, a package sample was perpendicularly cut with a one-sided razor blade to obtain its cross section. Two steps were involved in measuring the impedance profile: (1) A focused transducer $\left(37.4 \mathrm{MHz}, B D_{(-6 \mathrm{~dB})}=52.9 \mu \mathrm{m}\right)$ scanned a flat Plexiglas surface whose impedance value was known (reference echo). (2) The transducer scanned the package sample cross-section $(220-\mu \mathrm{m}$ thick) at $5-\mu \mathrm{m}$ step increments, with the beam axis parallel to the package sample planar surface (Fig. 4). The rf echo waveform at each scan location was compared with the reference rf echo waveform to determine the impedance. Due to the cross-section edges, it was necessary to correct the impedance values at both ends of the impedance profile.

Optical microscopic images of the package sample cross-section were obtained to observe microstructure features. Several measured sample impedance profiles along the sample cross-section thickness dimension were examined in order to have a quantitative impedance measurement. To characterize the package sample cross-section, the 2D impedance map was normalized to the maximal impedance value in the scan region and displayed as an acoustic image. In all these images and impedance measurements, the transducer was placed on

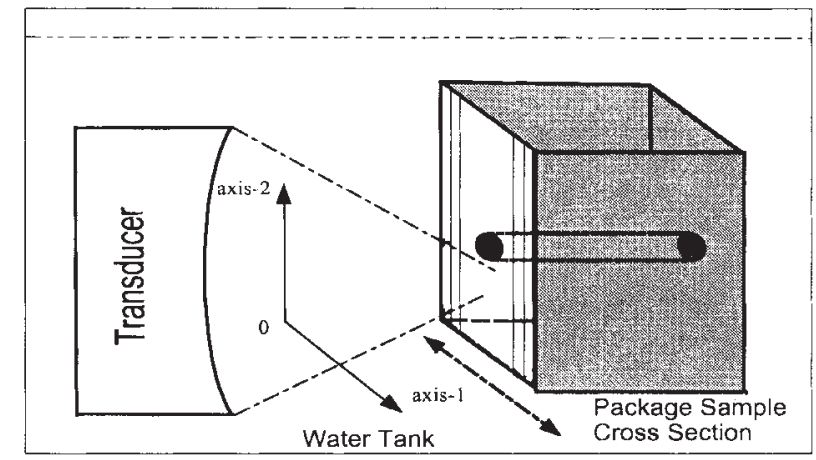

Fig. 4. Package sample cross-section impedance measurement.

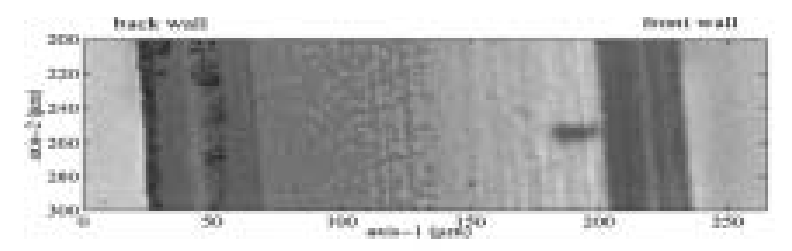

(a)

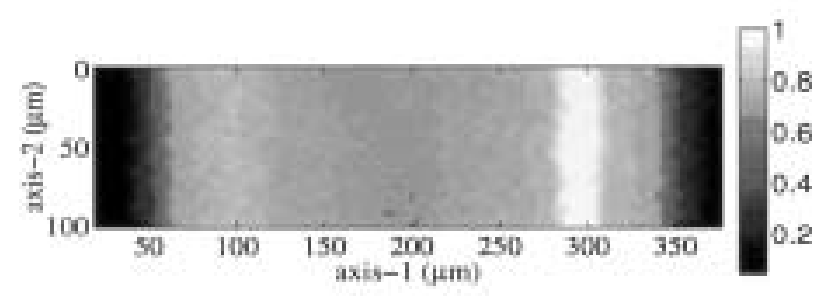

(b)

Fig. 5. (a) Optical microscopic image and (b) acoustic image of a package sample, without channel defect.

the right side, which made the sample right edge to be the package sample "front wall."

The microstructure of the package sample without channel defect was investigated to establish a generalized impedance profile for the intact region. The optical microscopic and acoustic images (Fig. 5) of the package sample cross-section were obtained. Both images showed clear sublayer features. They also demonstrated that the package sample had asymmetric acoustic properties. The vertical bright strip at $300 \mu \mathrm{m}$ in axis-1 [Fig. 5(b)] corresponded with the PVDC sublayer in one package film. This PVDC sublayer had the maximum impedance value in the acoustic image. The PVDC sublayer in the other package film should have had a corresponding vertical bright strip at $100 \mu \mathrm{m}$ in axis- 1 if the impedance profile were symmetric as the nominal values predicted (see Sec. 3.1). However, no obvious vertical bright strip appeared in the acoustic image at $100 \mu \mathrm{m}$ in axis-1. 
Two measured impedance profiles at two randomly chosen horizontal cuts in Fig. 5(a) are shown in Fig. 6. The maximum impedance occurred near the front wall and a dip appeared behind the maximum impedance sublayer. The impedance at the package sample back wall was less than that at the front wall.

Although identical in nominal impedance properties before heat sealing, the two binding films showed an asymmetric impedance profile after sealing (Figs. 5 and 6). The multiple reflections in the asymmetric sublayers would behave with more complexity than in the presumed symmetric sublayers.

The package samples with 75- $\mu$ m-diameter (Fig. 7) and 38- $\mu$ m-diameter (Fig. 8) water-filled channel defects were examined. The shapes of the channel

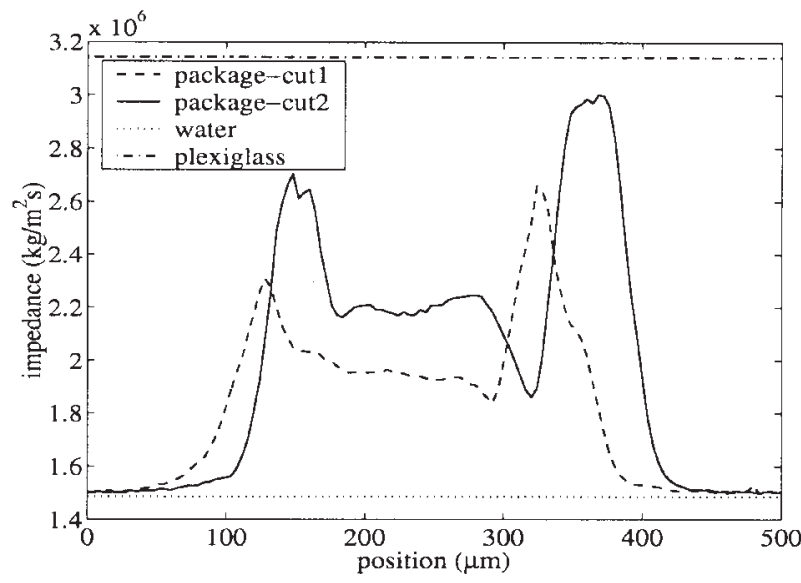

Fig. 6. Two impedance profiles of a package sample, without defect.

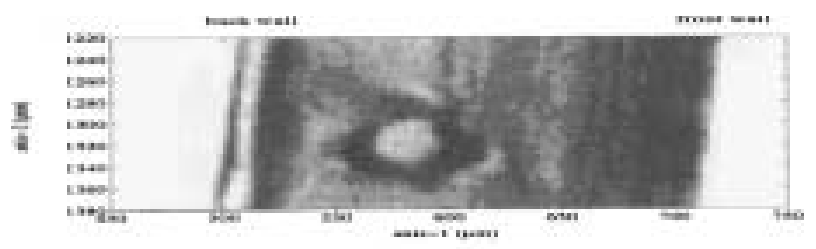

(a)

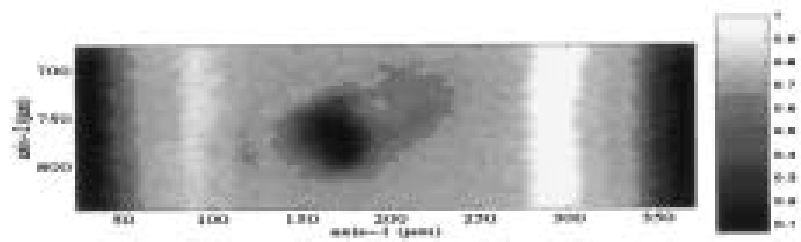

(b)

Fig. 7. (a) Optical microscopic image and (b) acoustic impedance image of a package sample containing a $75-\mu \mathrm{m}$-diameter water-filled channel.

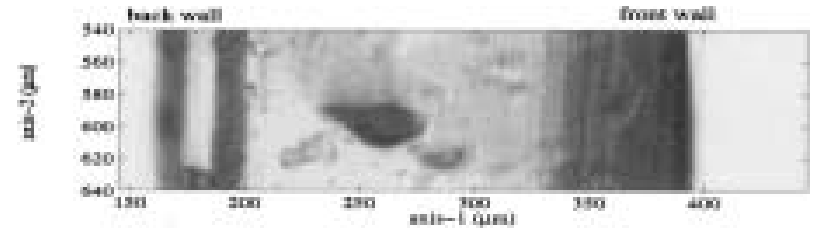

(a)

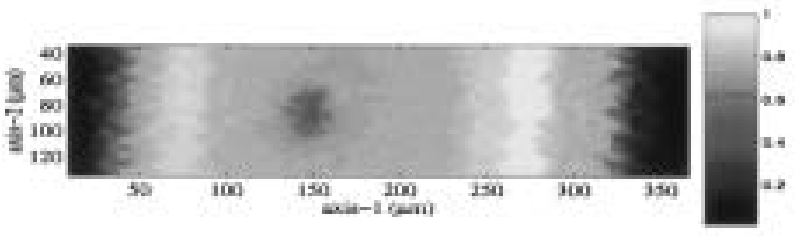

(b)

Fig. 8. (a) Optical microscopic image and (b) acoustic impedance image of a package sample containing a $38-\mu \mathrm{m}$-diameter water-filled channel.

defects were not perfect circles as that of the tungsten wire cross-section shape. The same observation was also made previously. ${ }^{(1,4)}$ The channel defects appeared to be elliptically shaped, and the channel defect contours were not smooth, demonstrating an impedance perturbation region surrounding the channel defect. The position of the channel defect was not exactly centered between package sample walls.

The impedance profiles in Figs. 5-8 span approximately $300 \mu \mathrm{m}$ while the sealed package samples are only $220-\mu \mathrm{m}$ thick. The thickness difference was introduced by the transducer focal beam spot $\left[B D_{(-6 \mathrm{~dB})}\right.$ $=52.9 \mu \mathrm{m}]$ at both edges. The finite beam spot size of the transducer limits the impedance profile measurement resolution. The measured acoustic impedance profiles are approximated impedance maps.

\section{CAUSE OF THE MICROSTRUCTURE FORMATION}

The experimental characterization of the package sample cross-section identified many microstructure features such as asymmetric impedance profiles, elliptic channel defect shapes, nonsmooth channel defect contours, and position shifts of channel defects. The elliptic shape might be caused by the material alteration induced by the applied static pressure from both sides of the sample (axis-1) during the heat sealing process, while there were no constraints from the other two sides (axis-2). The nonsmooth contour could be attributed to withdrawing of the wire manually after the sample had cooled. The heat conductivity of the tungsten wire is 
Table II. Material Thermal Properties

\begin{tabular}{lcccc}
\hline Material & $\begin{array}{c}\text { Conductivity } \\
h[\mathrm{~W} /(\mathrm{mK})]\end{array}$ & $\begin{array}{c}\text { Density } \\
\rho\left[\mathrm{kg} / \mathrm{m}^{3}\right]\end{array}$ & $\begin{array}{c}\text { Specific heat } \\
c_{\mathrm{v}}[\mathrm{J} /(\mathrm{kgK})]\end{array}$ & $\begin{array}{c}\text { Melting } \\
\text { point [K] }\end{array}$ \\
\hline $\begin{array}{l}\text { Polypropylene } \\
\begin{array}{l}\text { Polyvinylidene } \\
\text { chloride }\end{array}\end{array}$ & $\begin{array}{c}0.1-0.13 \\
\text { N/A }\end{array}$ & $\begin{array}{c}900 \\
\text { (use } 0.05)\end{array}$ & $\begin{array}{c}2000 \\
\text { N/A }\end{array}$ & 403 \\
$\begin{array}{l}\text { Polystyrene } \\
\text { Oriented nylon }\end{array}$ & $\begin{array}{c}\text { N/A } \\
\text { (at } 357.9 \mathrm{~K})\end{array}$ & 1050 & $\begin{array}{c}\text { (use } 0.157) \\
0.157\end{array}$ & N/A \\
Tungsten & $\begin{array}{l}179 \\
\text { (at } 273 \mathrm{~K})\end{array}$ & 19300 & $9.31 \times 10^{-7}$ & N/A \\
Rubber & 0.163 & 1150 & 2009 & 3653 \\
\hline
\end{tabular}

significantly greater than those of the package sample sublayer materials (Table II) so that the tungsten wire acted as a heat sink that attracted more heat flow in the defect region than in the intact region during the heating process.

The microstructure formation cause was investigated. The two sheets of plastic film were tightly clamped between one heating bar (with a flat nonstick coating) and one pressure bar (thick flat silicone rubber) during the sealing process (Fig. 3). Heat was transferred from the heating bar and reached the contacting surfaces of the two films, causing them to melt and merge together. The impedance values at the package sample back wall were always less than those at the front wall, and the back wall was the surface in contact with the heating bar (Fig. 3); this led to an intuitive guess that the single-sided heating during the sealing process caused the asymmetric impedance profile.

The heat-sealing process is a typical heat-conduction process. When molecular random motion exists and a temperature gradient is present, heat transfer occurs via the conductive process, which is described by the heat-conduction equation ${ }^{(23)}$ given by

$$
h \nabla^{2} \mathcal{T}+q=\rho c_{\mathrm{v}} \frac{\partial \mathcal{T}}{\partial t}
$$

where $\mathcal{T}$ is the temperature $[\mathcal{T}(x, y, z, t)], h$ is the thermal conductivity of the material, $\rho$ is the mass density, $c_{\nu}$ is the specific heat, and $q$ is the heat generation rate per unit volume. The operator $\nabla^{2}$ is the Laplacian.

Considering the package sample thickness (220 $\mu \mathrm{m})$ and the heating time (usually $1 \sim 2 \mathrm{~s}$ ), a transient thermal finite-element analysis (FEA) was performed using ANSYS (ANSYS Inc., Canonsburg, PA) to solve the heat conduction Eq. (4) in $2 \mathrm{D}$ and verify this conjecture.

The material properties $\left(\rho, c_{\nu}, h\right)$ are listed in Table II. Table II also lists the material melting points. The exact thermal conductivity and specific heat were unavailable for the polyvinylidene chloride used in the samples. Because the thermal analysis is not an exact quantitative analysis, these values are set by taking appropriate values from other similar material such as polystyrene. All the material properties in Table II are cited from Refs. 23-25.

The configuration of the thermal FEA is illustrated in Fig. 9(a). To make the simulation more realistic to the heat-sealing situation, a 2000- $\mu$ m-thick rubber layer was included to simulate the pressure bar that was in close contact with the top edge of sheet A in Fig. 3(a). The initial temperature was set at room temperature $(293 \mathrm{~K})$. The boundary condition on the bottom edge of sheet B was set as an incoming heat flux from the heating bar, representing an intimate contact between the heating bar and the package material. The circle in Fig. 9(a) was the 38- $\mu$ m-diameter tungsten wire cross section. The eight $\mathrm{Xs}$ were the designated temperature observation points (A, B, C, D, E, F, G, and H). Point A was on the bottom edge of sheet B. Point D was on the interface between sheets A and B. Point G was on the top edge of sheet A. Point $\mathrm{H}$ was on the top edge of the rubber layer.

A 24-VAC electrical current was applied on a metal strip of the heating bar. By electric-to-thermal energy conversion, heat was then emitted from the two flat surfaces of the metal strip, half going to the package sample material and the other half going to the opposite direction. The measured electrical resistance of the metal strip was $1 \Omega$. The electric-to-thermal energy conversion is assumed to be $50 \%$. (It is difficult to quantitatively estimate the actual conversion percentage. The $50 \%$ estimation considers both the energy conversion efficiency and the energy loss in heat transfer from the metal strip to the package material.) The total thermal power emitted from the heating bar is thus $Q=50 \% \times 50 \% \times U^{2} / R=0.25 \times(24 \times 24 / 1)$ $=144 \mathrm{~W}$, where $U=24 \mathrm{~V}$ was the root-mean-square voltage applied on the metal strip ${ }^{(26)}$. The area of the heating bar was $0.4 \mathrm{~m}$ by $0.08 \mathrm{~m}$. Thus, the heat flux would be $Q / A=144 /(0.4 \times 0.08)=45 \mathrm{~kW} / \mathrm{m}^{2}$. The heat flux had a constant value of $45 \mathrm{~kW} / \mathrm{m}^{2}$ from 0.1 to 1.6 seconds, representing the heating phase. The heat flux was zero from 1.6 to 3.0 seconds, representing the cooling phase.

The thermal FEA was conducted on both the package samples with and without tungsten wire. Figure 9(b) shows that the temperature changed at different rates with different package sample depths. The temperature difference of about $150 \mathrm{~K}$ along the $220-\mu \mathrm{m}$ sample thickness was demonstrated in the single-sided heat-sealing process. The maximum temperatures of the curves 


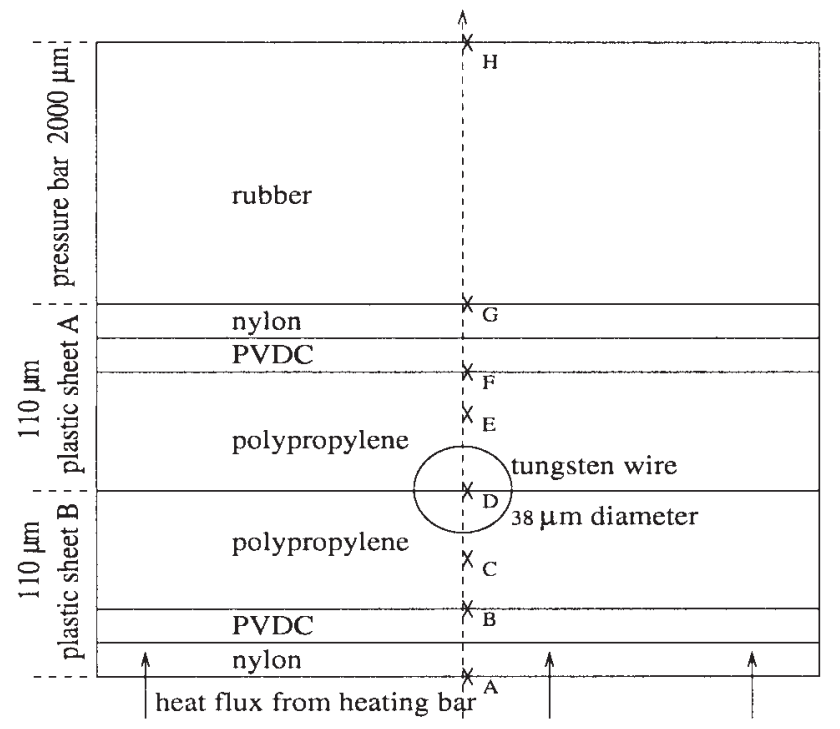

(a)
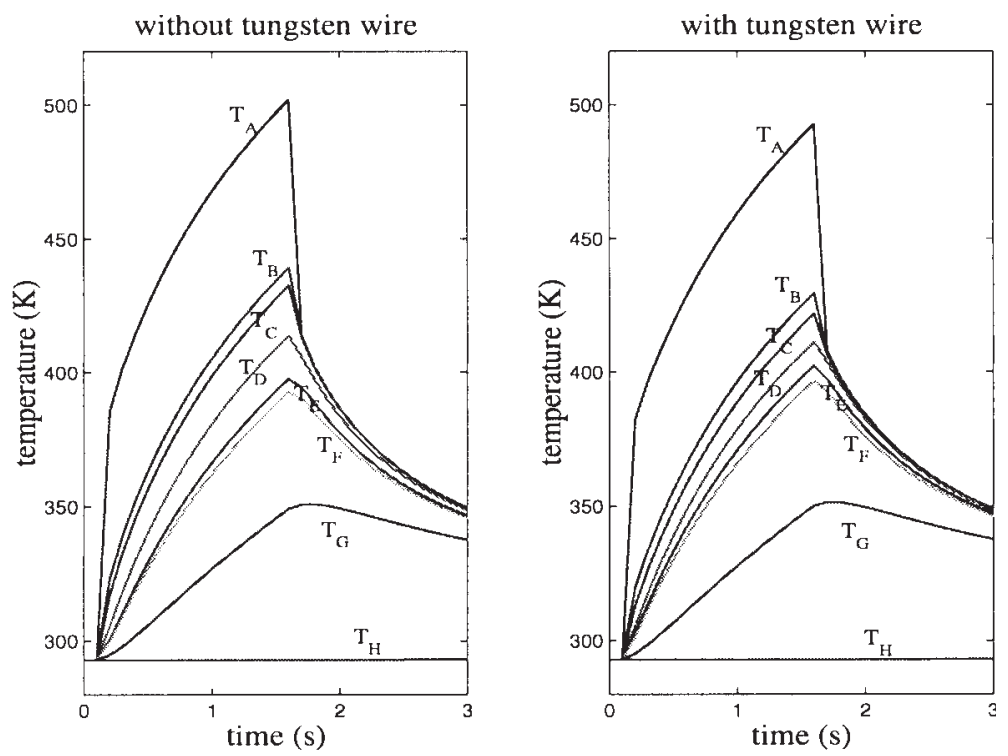

(b)

Fig. 9. (a) ANSYS heat conduction FEA configuration; (b) temperature-time history inside the package sample during the heat-sealing process. The heating starts at $t=0.1 \mathrm{~s}$ and ends at $t=1.6 \mathrm{~s}$.

in Fig. 9(b) were inversely proportional to their depths from the bottom edge of sheet $\mathrm{B}$. The temperatures in sheet $\mathrm{B}\left(T_{\mathrm{B}}, T_{\mathrm{C}}\right.$, and $\left.T_{\mathrm{D}}\right)$ exceeded the material melting point at the end of the heating phase whereas the temperatures in sheet $\mathrm{A}\left(T_{\mathrm{E}}, T_{\mathrm{F}}\right.$, and $\left.T_{\mathrm{G}}\right)$ did not exceed the material melting point at the end of the heating phase. The two sheets would have melted at their contacting surfaces but experienced different temperature-time history. The actual package seal condition would depend on the heat flux and the heating time.

The introduction of the tungsten wire not only reduced the maximum temperature shown by each temperature curve, but also caused the curves around the tungsten wire region $\left[T_{\mathrm{C}}\right.$ and $T_{\mathrm{E}}$ in Fig. 9(b)] to converge more closely than that of the no tungsten wire case. It showed that the tungsten wire functioned as a 
heat sink to attract a large amount of heat to its surrounding region. The intensive heat flow and the presence of the tungsten wire in this region could possibly alter the material properties around the tungsten wire.

Further experiments were done to investigate the cause of the microstructure formation. Figure 10 shows the measured package sample cross-section impedance profiles for four cases. Figure 10(a) was the profile for a single-sheet package film. The impedance profile showed a maximum impedance sublayer (PVDC sublayer). After sealing the two package film sheets [A and B in FIG. 3(a)], the PVDC sublayer near the top edge of sheet $\mathrm{A}$ had the maximum impedance, but the other PVDC sublayer impedance decreased [Fig. 10(b)]. The impedance profile was asymmetric (see description for Fig. 6). The sealed package sample underwent the second sealing process with the upper side down [flip A and B in FIG. 3(a)] after the first sealing was done. The measured impedance profile [Fig. 10(c)] regained symmetry, and the sharp dips after the PVDC sublayer became less striking. The double sealing made the impedance profile return to symmetry because each side of the sample experienced one heating process, exerting a balanced impact on the impedance profile. Furthermore, the third heat-sealing process was done after the double sealing. This time the impedance profile [Fig. 10(d)] became irregular because of the overheating after the triple-sealing process. However, the asymmetric pattern appeared again, with the maximum occurring near the front wall.

These experiments and the FEA analysis support the intuitive conjecture about the formation cause of the package sample microstructure. It is the singlesided heating that causes the asymmetric impedance profile for the package sample. The unbalanced heat conduction induces asymmetric property changes during the sealing process.

\section{NUMERICAL RESULTS AND DISCUSSION}

\subsection{Generalized Impedance Model}

A generalized impedance profile was proposed based on the microstructure characterization of the package sample. The generalized impedance model (Fig. 11) includes features for intact package-sealing region and channel defect region.

For the intact package-sealing region, the model considers (1) asymmetric impedance profile across the six sublayers whose thicknesses were depicted in

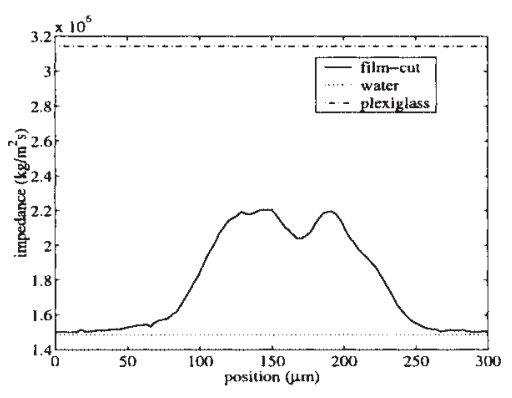

(a)

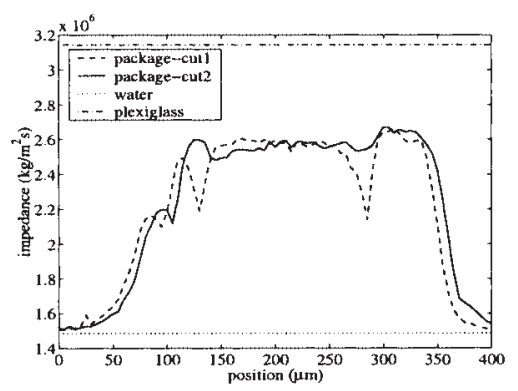

(c)

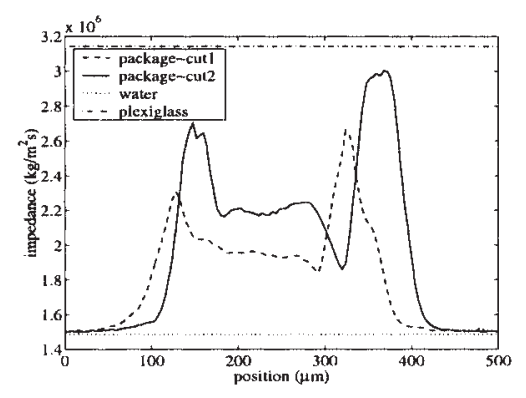

(b)

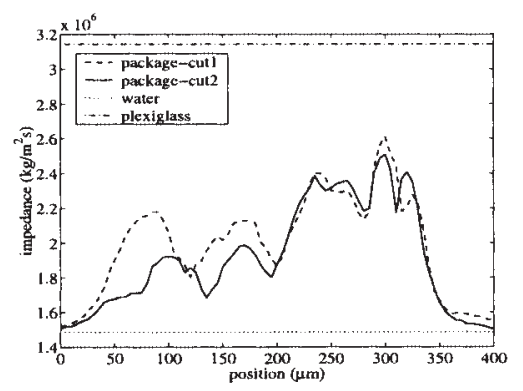

(d)

Fig. 10. Package sample impedance profiles: (a) single sheet, no sealing; (b) double sheets, sealed once; (c) double sheets, sealed twice from both sides; (d) double sheets, sealed three times with two on one side and one on the other side. 


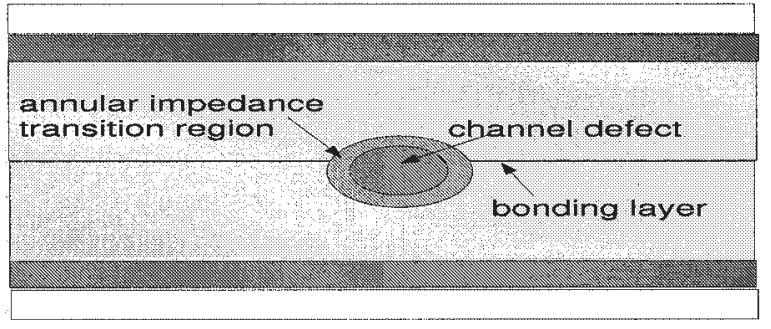

Fig. 11. Proposed package sample microstructure impedance model The bottom edge corresponds with the contacting surface with heating bar during heat-sealing process.

Sec. 3.1, (2) bonding sublayer (impedance deviation sublayer) between the two plastic films, and (3) frequencyindependent weak attenuation inside the packaging material. The establishment of a generalized impedance model for the intact region is a prerequisite before building up the model for the channel defect region.

For the channel defect region, the model considers (1) elliptically shaped channel defect with a nonsmooth contour, (2) annular impedance transition region surrounding the channel defect, and (3) channel defect position shift along the sample thickness dimension. These features were observed in Sec. 3.2.

\subsection{Simulated Echoes}

By implementing the proposed impedance model, we hope to establish a generalized model that would produce the simulated rf echo waveforms with high similarity to the measured echoes (Fig. 1) so that the above impedance model could be examined in a numerical approach.

Four simulations were done for four different impedance maps. For the control case (case 1), the impedance profile was symmetric. Case 2 used an asymmetric impedance profile, without any defect. Case 2 was used to test the generalized impedance model for the intact region. Case 3 added a water-filled channel defect to the impedance map of case 2. Furthermore, case 4 added the annular impedance region to the impedance map of case 3 . The difference between cases 3 and 4 was the annular impedance region in the impedance map. The four impedance profiles along the sample thickness dimension in the proposed impedance model are shown in Fig. 12(a). Figure 12(b) shows the simulated impedance map of case 4 , which is a package sample with a $38-\mu \mathrm{m}$-diameter channel defect and the annular impedance region. The ultrasonic beam was incident from the right in both figures.

The channel defect was modeled as an ellipse with a 38- $\mu \mathrm{m}$ major axis and a 30- $\mu \mathrm{m}$ minor axis. The minor axis was parallel with the beam axis. The defect was filled with water $\left(\rho=1000 \mathrm{~kg} / \mathrm{m}^{3}, c=1485 \mathrm{~m} / \mathrm{s}\right)$ and there was a $20-\mu \mathrm{m}$-thick surrounding annular region whose impedance was greater $\left(0.2 \sim 0.6 \mathrm{Mkg} / \mathrm{m}^{2} \mathrm{~s}\right)$ than that of the intact region. The nonsmooth channel contour was constructed by inwardly or outwardly shifting a small position offset between 0 and $6 \mu \mathrm{m}$ randomly on the smooth elliptic contour. The channel defect had a $35-\mu \mathrm{m}$ position shift toward the package sample front wall. The package material attenuation coefficient was set as $20 \mathrm{~dB} / \mathrm{mm}$, which was a measured value from a

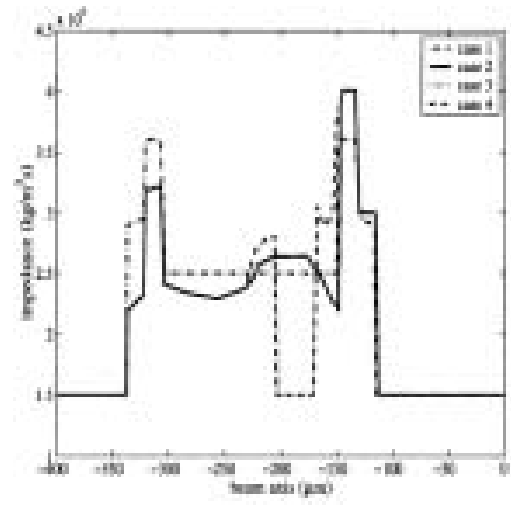

(a)

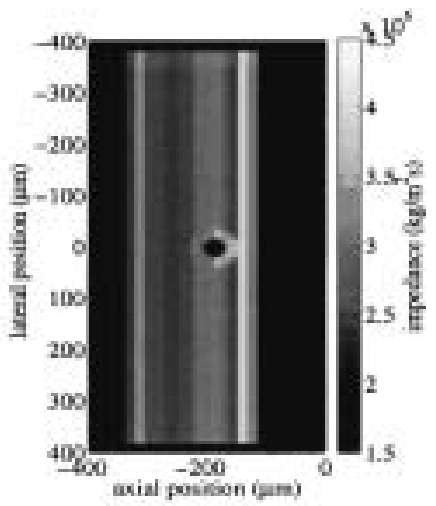

(b)

Fig. 12. Generalized impedance model: (a) impedance profiles for the four cases (case 1, symmetric impedance without defect; case 2, asymmetric impedance without defect; case 3, asymmetric impedance with a water-filled channel; case 4 , asymmetric impedance with a water-filled channel and an annular impedance region); (b) impedance map of the defect sample for case 4. 
pulse-echo insertion-loss measurement of the package sample at $17.3 \mathrm{MHz}$. The attenuation coefficient in the annular region is unavailable. In the annular region, the attenuation coefficient was assumed to be $40 \mathrm{~dB} / \mathrm{mm}$. Implementing frequency-dependent attenuation coefficients in FDTD simulation is a complicated task. Because the annular region is very small, and the transducer bandwidth is about $8 \mathrm{MHz}$, both attenuation coefficients were assumed to be frequency independent without losing too much accuracy while keeping the FDTD simulation simple.

The simulated rf echo waveforms for the four cases were obtained. Both echo waveforms and their frequency-domain amplitude are plotted for comparison. Figure 13 presents the comparison between the measured echo from intact region and the simulated echoes from the symmetric (case 1) and the asymmetric (case 2) impedance profiles. The simulated echo in case 2 resembled the measured echo better than that from case 1 did. Figure 14 presents the comparison between the measured echo from the defect and the simulated echoes from the asymmetric impedance with water-filled channel only (case 3 ) and with both waterfilled channel and annular impedance region (case 4). Adding an annular impedance region affected the echo waveform and further simulated the pulse-echo detection better than would have been the case if only a channel were present.

The normalized cross-correlation coefficient $R$ between the simulated echo waveform and the measured echo waveform was calculated as a quantitative index of similarity between them. $\Delta$ BAI was also calculated for the experiment measurement and for cases 3 and 4 . The $\triangle \mathrm{BAI}$ is defined as $\left|B A I_{\text {flawed }}-B A I_{\text {intact }}\right| / B A I_{\text {intact }}$,
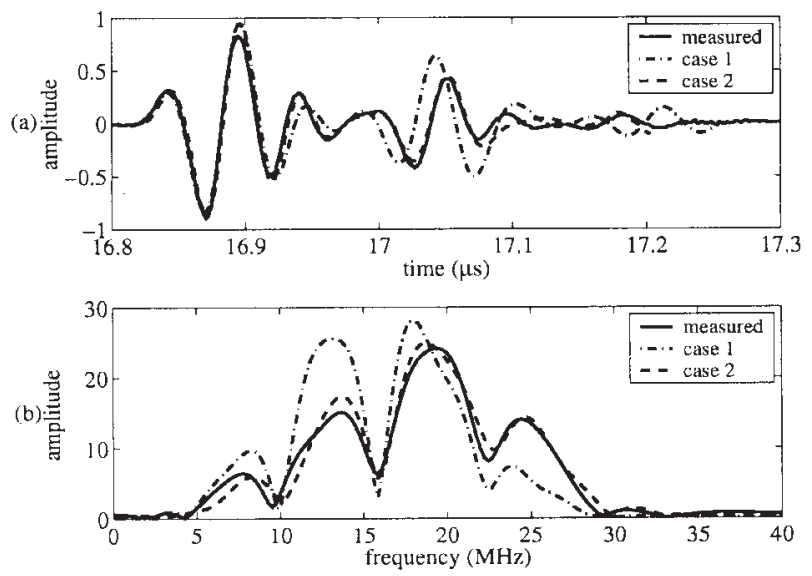

Fig. 13. Comparison between the measured echo waveform and the simulated rf echoes for cases 1 and 2: (a) time-domain waveforms; (b) frequency-domain amplitude.
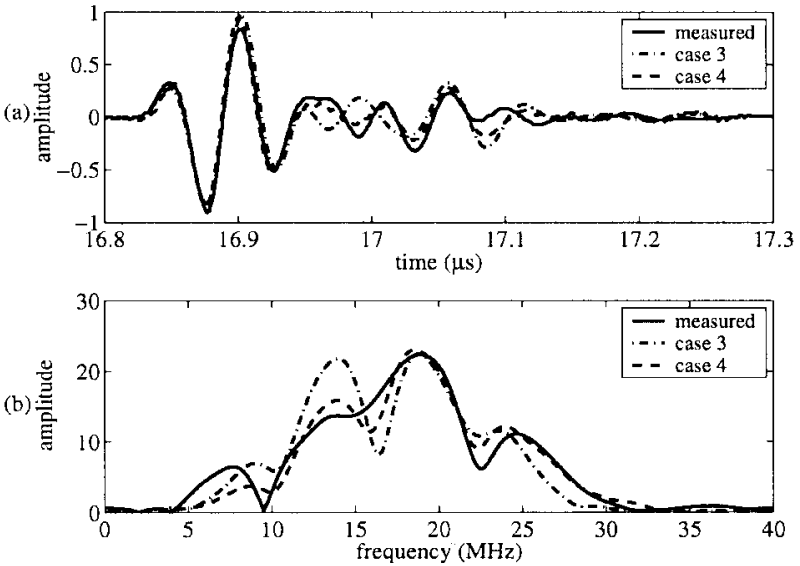

Fig. 14. Comparison between the measured echo waveform and the simulated rf echoes for cases 3 and 4: (a) time-domain waveforms; (b) frequency-domain amplitude.

Table III. Normalized Cross-Correlation Coefficients and $\Delta \mathrm{BAI}$ Values

\begin{tabular}{lccccc}
\hline & Case 1 & Case 2 & Case 3 & Case 4 & Experiment \\
\hline$R$ & $80.4 \%$ & $97.7 \%$ & $88.4 \%$ & $95.7 \%$ & N/A \\
Note for $R$ & Against the & Against the & \\
& measured echo & measured echo & \\
& from intact region & from defect & \\
$\Delta$ BAI & N/A & N/A & 0.0305 & 0.1167 & 0.0901 \\
\hline
\end{tabular}

which is the BAI-value contrast between defective and intact regions. $\triangle \mathrm{BAI}$ indicates the image contrast in a BAI-mode image (see Sec. 1 for BAI-mode images). Table III summarizes the $R$ values and the $\Delta$ BAI values. Case 2 has a higher $R$ value $(97.7 \%)$ than that of case $1(80.4 \%)$ when compared with the measured echo from the intact region. Case 4 has a higher $R$ value $(95.7 \%)$ than that of case $3(88.4 \%)$ when compared with the measured echo from the channel defect region. The $\Delta$ BAI value for case $4(0.1167)$ is closer to that of the experiment (0.0901) than that of case $3(0.0305)$. These results provide quantitative support for the hypotheses of the asymmetric impedance profile and the annular impedance region. The simulated results match with the experimental results very well for the proposed impedance model.

\subsection{Discussion}

The asymmetric impedance profile, along with the uniform attenuation in the material, was used to determine the rf echo waveform in the intact region. The echo amplitude was not zero between the two echoes 
from package sample front and back walls because the corresponding impedance in this region was not uniform. The impedance variation caused reflections.

The channel defect alone could not produce a significant echo waveform variation in the time interval between the two echoes from the front and back walls of package sample. The insertion of the tungsten wire during the heat-sealing process took up the volume originally occupied by the package material. Consequently, the annular region was produced by material alteration during the heat-sealing process. Because of the material alteration, this annular region tended to be more solid-like with impedance value higher than that of its surrounding medium. The impedance difference between the annular region and the defect region (water) increased. As a result, the rf echo waveform had a significant amplitude variation in the channel defect region due to the increased impedance difference.

The shape and position of the channel defect also played an important role in determining the rf echo waveform. If the defect shape were smooth and round, caustics would occur because the perturbation of the smooth scatterer acted as a focal lens to the incident acoustic rays. The caustics might have then produced an enhancement of the echo amplitude from behind the smooth scatterer, depending on the shape of the curvature. If the distance happened to be inside the package sample, then the echo amplitude would have increased behind the defect. However, the measured echo from the channel defect showed that the echo amplitude decreased from behind the defect when compared with the echo from the intact region. The nonsmooth defect contour disrupted the condition to form caustics by randomly diverting the acoustic rays into different directions around the defect.

Because of the limited understanding of microstructure information, several assumptions had to be made to estimate the details of the package sample. For example, the acoustic impedance measurement provided only an approximate impedance map due to the transducer lateral resolution $\left(B D_{-6 \mathrm{~dB}}=52.9 \mu \mathrm{m}\right)$. It is a very challenging task to model every aspect of the pulse-echo subwavelength defect detection process.

\section{CONCLUSIONS}

A theoretical modeling study was undertaken to investigate the pulse-echo subwavelength defect detection mechanism through both experimental characterization and numerical modeling studies. The impedance profiles in the flexible package samples were asymmetric due to the single-sided heat-sealing process. A generalized impedance model of the package sample with a channel defect was proposed and evaluated through numerical simulation in this study. The simulation results verified the model and explained the origin of the rf echo amplitude variations between the echoes from the intact and channel defect regions. The asymmetric impedance profile of the package sample, along with the uniform attenuation, caused the amplitude variations of the echo waveform from the intact region. Material alteration around the channel defect effectively enlarged the defective area, which increased the defect detectability during the pulse-echo detection process. This alteration of the structure's material and the apparent subsequent enhancement of the BAI values indicate several intriguing possibilities. The first of these is that the detection of naturally occurring defects without the material changes, and subsequent impedance difference to provide a strong backscattering interface, would be less certain. It also indicates that there is further work necessary in the understanding of the nature of the material alterations that are apparently occurring in the polymer structure and morphology as a result of the formation of these artificially created defects. Understanding the fundamental pulse-echo subwavelength defect detection mechanism has thus improved our knowledge in material characterization and nondestructive evaluation using ultrasonic pulse-echo techniques.

\section{ACKNOWLEDGMENTS}

This work was supported by the Illinois Council on Food and Agricultural Research (C-FAR) Competitive Grants Program. The authors would like to thank Dr. James F. Zachary and Mr. James P. Blue, Jr., for their professional assistance with the optical microscopic image and Dr. Michael L. Oelze for his helpful discussion with the acoustic impedance measurement experiment.

\section{REFERENCES}

1. K. Raum, A. Ozguler, S. A. Morris, and W. D. O'Brien, Jr., "Channel defect detection in shelf-stable food packages using high-frequency pulse-echo imaging," IEEE Trans. Ultrason., Ferroelect., Freq. Contr. 45, pp. 30-40 (1998).

2. C. H. Frzaier, Q. Tian, A. Ozguler, S. A. Morris, and W. D. O'Brien, Jr., "High contrast ultrasound images of defects in food package seals," IEEE Trans. Ultrason., Ferroelect., Freq. Contr. 47, pp. 530-539 (2000). 
3. Ozguler, S. A. Morris, and W. D. O'Brien, Jr., "Evaluation of defects in seal region of food packages using the backscattered amplitude integral (BAI) technique," in 1997 IEEE Ultrasonics Symposium Proceedings, edited by S. C. Schneider, M. Levy, and B. R. McAovy, vol 1, pp. 689-692, (Toronto, Canada, 1997).

4. Ozguler, S. A. Morris, and W. D. O’Brien, Jr., "Evaluation of defects in the seal region of food packages using the ultrasonic contrast descriptor, $\Delta \mathrm{BAI}, "$ Packaging Technol. Sci. 12, pp. 161-171 (1999).

5. X. Yin, S. A. Morris, and W. D. O'Brien, Jr., "Investigation of spatial sampling resolution of the real-time ultrasound pulse-echo BAI-mode imaging technique," in 2001 IEEE Ultrasonics Symposium Proceedings, edited by S. C. Schneider, M. Levy, and B. R. McAovy, vol 1, pp. 729-732, (Atlanta, GA, 2001).

6. X. Yin, S. A. Morris, and W. D. O'Brien, Jr., "Experimenta spatial sampling study of the real-time ultrasonic pulse-echo BAI-mode imaging technique," IEEE Trans. Ultrason., Ferroelect., Freq. Contr. 50, pp. 428-440 (2003).

7. A. D. Pierce, Acoustics: An Introduction to Its Physical Principles and Applications (The Acoustical Society of America, Woodbury, NY, 1989).

8. K. S. Yee, "Numerical solution of initial boundary value problems involving Maxwell's equations in isotropic media," IEEE Trans. Antennas and Propagation 14, pp. 302-307 (1966).

9. P. Berenger, "A perfectly matched layer for the absorption of electromagnetic waves," J. Comput. Phys. 114, pp. 185-200 (1994).

10. X. Yuan, D. Borup, J. W. Wiskin, M. Berggren, R. Eidens, and S. A. Johnson, "Formulation and validation of Berenger's PML absorbing boundary for the FDTD simulation of acoustic scattering," IEEE Trans. Ultrason., Ferroelect., Freq. Contr. 44, pp. 816-822 (1997).

11. H. Chang and R. M. D'angelo, "Experimental verification of finite-difference model for ultrasonic evaluation of complex structures," in 1990 IEEE Ultrasonics Symposium Proceedings, edited by B. R. McAvoy, vol 2, pp. 965-969, (Honolulu, HI, 1990).

12. P. H. Johnston and E. I. Madaras, "Modeling the pulse-echo response of a two-dimensional phase-insensitive array for NDE of layered media," 1988 IEEE Ultrasonics Symposium Proceedings, edited by B. R. McAvoy, vol 2, pp. 1021-1025, (Chicago, IL, 1988).
13. R. Stacey and J. P. Weight, "Ultrasonic pulse-echo responses from targets in solid media using finite difference methods," IEE Proceedings-A 140, pp. 303-316, (1993).

14. L. P. Martin, D. H. Chambers, and G. H. Thomas, "Experimental and simulated ultrasonic characterization of complex damage in fused silica," IEEE Trans. Ultrason., Ferroelect., Freq. Contr. 49, pp. 255-265, (2002).

15. K. L. Shlager and J. B. Schneider, "A selective survey of the finite-difference time-domain literature," IEEE Antennas and Propagation Magazine 37, pp. 39-57, (1995).

16. A. Taflove, "Review of the Formulation and Applications of the Finite-Difference Time-Domain Method of Numerical Modeling of Electromagnetic Wave Interactions with Arbitrary Structures," Wave Motion 10, pp. 547-582, (1988).

17. W. Manry Jr., S. L. Broschat and J. B. Schneider, "Higher-order FDTD methods for large problems," J. Appl. Comput. Electromag. Soc. 10, pp. 17-29, (1995).

18. J. A. Jensen, User's guide for the FIELD-II program Available: http://www.es.oersted.dtu.dk/staff/jaj/field/information/users guide.pdf.

19. V. A. Dumane and P. M. Shanker, "Use of frequency diversity and Nakagami statistics in ultrasonic tissue characterization," IEEE Trans. Ultrason., Ferroelect., Freq. Contr. 48, pp. 1139-1146 (2001).

20. R. Lerch, H. Landes and H. T. Kaarmann, "Finite element modeling of the pulse-echo behavior of ultrasound transducers," in 1994 IEEE Ultrasonics Symposium Proceedings, edited by S. C. Schneider, M. Levy, B. R. McAovy, vol. 2, pp. 1021-1025 (Cannes, France, 1994).

21. E. Skudrzyk, The Foundations of Acoustics (Springer-Verlag, New York, NY, 1971)

22. K. Raum and W. D. O'Brien, Jr., "Pulse-echo field distribution measurement technique for high-frequency ultrasound sources," IEEE Trans. Ultrason., Ferroelect., Freq. Contr. 44, pp. 810-815 (1997)

23. W. S. Janna, Engineering Heat Transfer, 2nd ed. (CRC Press LLC, Boca Raton, FL, 2000)

24. T. P. R. C. of Purdue University, Thermophysical Properties of Matter (IFI/Plenum, New York, 1970-1979).

25. Material properties online database. Available: http:// www.matweb.com. 